\title{
BIBLIOGRAFIA
}

\section{Bibliografía de la novela publicada en Cuba, y en el extranjero por cubanos, desde 1959 hasta 1965}

I. Abreu, José Vicente. Se llamabra S.N. Habana: Editorial Venceremos, I965.

2. Agostini, Víctor. Dos viajes; novela. La Habana: Editorial Nacional de Cuba, Ediciones R, I965.

3. Aguilar Derpich, Juan. "El Majos" a El pérfído Julián. La Habana, I963.

4. - Se alquilan cuartos amweblados. Habana, 1962.

5. Aguililla, Araceli de. Primaros recuerdos. Habana, I96.

6. Alonso, Dora. Tierra inerme; novela. r. ed. Habana: Casa de las Américas, I96r. (Colección concurso literatio hispanoamericano).

7. —. Tierwa inerme. La Habana: Editorial Nacional de Cuba, r965. (Colección bolsilibros).

8. Amador Martz, Ana Maria. Alma bueca; novela. Habana: Editorial San Antonio, I960.

9. Arcocha, Juan. Los muertos andan solos; novela. r. ed. Habana: Ediciones $\mathrm{R}$, I962.

ıo. Arenal, Humberto. El sol a plomo. New York: Las Americas Publishing Co., I9,59.

Ir. - - El sol a plomo; novela. 2. ed. Habana: Cruzada Latinoamericana de difusión cultural, I959. (Comprensión de Cuba, 5).

r2. - - The sun beats down; a novella of the Cuban revolution. Trans- 
lated from the Spanish, by Joseph M. Bernstein. New York: Hill and 'Wang, I959.

I3. Armenteros, Emma. Guccoma; novela. Fotografías por Lezcano. Habana: Talleres Guamá, I964.

I4. Becali, Ramón. Los dioses mentigos. La Habana, I965.

I5. Becerra Ortega, José. La novena estacionn; novela basada en hechos reales de la revolución. Habana: Impr. "El Siglo XX", I959.

I6. Benet y Castellón, Eduardo. Birín; novela. Santa Clara: Universidad Central de las Villas, Dirección de Publicaciones, I962. (Nuevo plan de publicaciones no. 37).

I7. Bravo Adams, Caridad. Flor salvaje. México: Editorial Diana, I96o.

I8. - Lo que tú callaste; novela. México: Editorial Diana, I96r.

I9. - - Reina sin corona. México: Editorial Diana, 196r.

20. - Senda die rencor. r. ed. México: Editorial Diana, r962.

21. Cabrera Infante, Guillermo. Vistá del amanecer en trópico. I965. (Biblioteca breve). Premio Joan Petit.

22. Camus, Emilio Fernández. Camizros llenos de borrascas. Madrid: Gráficas Orbe, I962.

23. Carpentier, Alejo. Explasion in a catbjedral; a novel. Translated, by John Sturrock. Ist. American ed. Boston: Little Brown, I963. Traducción de: El siglo de las luces.

24. - L Le siècle des lumières. Traduit de l'espagnol, par Reneé L. F. Durand. Prèf. de Jean Blanzat. Paris: Gallimard, I962. (La croix du Sud). Traducción de: El siglo de las ludes.

25. - - El siglo de las luces. México: Cía. General de Ediciones, I962. (Ideas, letras, vidas).

26. - El siglo de las luces. La Habana: Ediciones $\mathrm{R}, 1963$.

27. Carrión, Miguel de. La esfinge; novella. Prólogo de Mario Parajón. Habana: Comisión Nacional Cubana de la UNESCO, I96r.

28. Céspedes, Augusto. Metal del diablo. La Habana: Casa de las Américas, 196 .

29. Cuesta y Cuesta, Alfonso. Los hijos. Habana: Casa de las Américas, I963. (Colección concurso).

30. Desnoes, Edmundo. El calaclismo. La Habana: Editorial Nacional de Cuba, Ediciones R, I965.

31. - Mamorias del subdesarrollo; novela. La Habana: Editorial Nacional de Cuba, r965. (Ediciones Unión, Colección contemporáneos).

32. - No bay problemas; novela. Habana: Ediciones R, I96I.

33. - No bay problemas. La Habana: Ediciones R, 1964. 
34. Desnoes, Edmundo. Na bay problemias. I. ed. Traducida al ruso por Boris Polevoi. Moscú, I963.

35. Díaz Versón, Salvador. Ya el mundo oscurece; novela histórica de la revolución cubana. México: Ediciones Botas, I96r.

36. Enríquez, Carlos. La feria de Guaicanama; novela. Habana: Ministerio de Educación, Departamento Nacional de Culltura, I960. (Los contemporáneos).

37. - - Tilin Gariáa novela. La Habana: Ministerio de Educación, Dirección de Cultura, I960. (Los contemporáneos).

38. - La vuelta de Chencho; novela. Habana: Ministerio de Educación, Dirección de Cultura, I960. (Los contemporáneos).

39. Feijóo, Samuel. Juan Quinquin en Pueblo Mocho. Santa Clara: Universidad Central de las Villas, Dirección de publicaciones, I964. (Nuevo plan de publicaciones, no. 54).

40. - Tumbaga. Santa Clara: Universidad Central de las Villas, I964. (Nuevo plan de publicaciones no. 58 )

4I. García, Daura Olema. Maestra valuntaria. La Habana: Casa de las Américas, I962.

42. Gaviria, Rafael Humberto. El atentado; novela. La Habana: Tierra Nueva, I96r.

43. González Cascorro, Raúl. Concentración pública. La Habana, I964. (Ediciones Unión/novela).

44. Gravina, Alfredo D. Fronteras al viento. La Habana: Impr. Nacional de Cuba, 1962. (Biblioteca del pueblo).

45. Ibargüengoitia, Jorge. Las relámpagios de agosto. La Habana: Editora Nacional de Cuba, I964. (Colección concurso).

46. Leante, César. El perseguido. La Habana: Editorial Nacional de Cuba, Ediciones R, 1965 .

47. López Nusa, Leonel. Tabaco; novela. Santa Clara: Universidad Central de las Villas, Dirección de publicaciones, r963. (Nuevo plan de publicaciones, no. 43).

48. Lorenzo Fuentes, José. Magucraya arriba. Santa Clara: Universidad Central de las Villas, Dirección de Publicaciones, 1963. (Nuevo plan de publicaciones, no. 47).

49. - - El sol ese enemigo. La Habana: Ediciones R, I962-I963.

50. Lu-Sin. Novelas escogidas. Traducción de Luis Enrique Delgado. La Habana: Impr. Nacional de Cuba, r96r. (Obras maestras).

5I. Macau, Miguel Angel. $Y$ se salvaba el amor; novela. La Habana: Talleres Tipográficos P. Fernández, I959. 
52. Manet, Eduardo. Al borde de la orilla. París: Ediciones Juillard, 1963.

53. Masota, Luis. María ilusión. La Habana: Distribuidora Antillana de Librería, r96r. (Colección Diali).

54. Navarto, Noel. Los dias de nuestra angustia. La Habana: Ediciones R, 1962 .

55. Otero, Lisandro. La situación. La Habana: Casa de las Américas, I963. (Colección concurso literatio hispanoamericano). Premio de novela, Casa de las Américas.

56. Perera, Hilda. Mañana es 26. La Habana: Lázaro Hnos., I960.

57. Pérez, Rafael Alcides. Brigada 2506. La Habana, I965.

58. Piñeiro, Abelardo. El descanso. La Habana: Unión de Escritores y Artistas de Cuba, I962. (Edición Unión/novela).

59. Piñera, Virgilio. Pelqueñas maniobras. La Habana: Ediciones R, I963.

6o. Pogolotti, Marcelo. El caserón del Cerro. Santa Clara: Universidad Central de las Villas, I96I. (Dirección de publicaciones, no. 27).

6r. Rivero Collado, Andrés. Enterrado vivo; novela. México: Editorial Dinamismo, 1960.

62. Rodriguez Herrera, Mariano. Después de la Z. La Habana: Ediciones $\mathrm{R}$, I964.

63. Sánchez Torrentó, Eugenio. Francisco Manduley; la historia de un pescador de ranas. Miami: Service offsett, I965.

64. Sarduy, Severo. Gestos. Barcelona: Seix Barral, 1963.

65. - Gestos. París: Editorial Du Seuil, 1963.

66. Sarusky, Jaime. La brisquedd; novela. I. ed. La Habana: Ediciones R, I962.

67. —. La búsquedda. 2 ed. La Habana: Ediciones R, I962.

68. Soler Puig, José. Bertillón 166. La Habana: Ministerio de Educación, 1960.

69. —- El derrambe; novela. Santiago de Cuba: Editora del Consejo Nacional de Universidades, Universidad de Oriente, 1964.

70. - En el año de eneyo. La Habana: Unión de Escritores y Artistas de Cuba, 1963. (Ediciones Unión-novela).

7I. Suárez Solís, Rafael. Un puebro donde no padaba naddat; novela del tiempo quieto. Santa Clara: Universidad Central de las Villas, 1962. (Dirección de publicaciones, no. 34).

72. Tellado, Corín. El cambió mi vida. La Habana: Distribución Antillana de Librería, I96r. (Colección Diali).

73. - -...Yo no soy asî... La Habana: Distribuidora Antillana de Librería, I96r. (Colección Diali). 
74. Tejera, Nivaria. El barranco. Santa Clara: Universidad Central de las Villas, Departamento de Relaciones Culturales, I959. (Publicaciones, no. 13 ).

75. Torriente Brau, Pablo de la. Aveinturas del soldado desconocido cubano; novela. Inicial de Raúl Roa. La Habana: Publicaciones del gobierno Provincial Revolucionario de La Habana, Departamento de Cultura, I960.

76. Vieta, Ezequiel. Vivir en Candonga. La Habana: Unión de Escritores y Artistas de Cuba, 1965. Premio Nacional de Novela "Cirilo Villaverde". 



\section{Bibliografía de la novela reimpresa en Cuba desde 1959 hasta 1965}

I. Asturias, Miguel Angel. El señor presidente. Habana: Ediciones La Tertulia, I960. (Biblioteca básica de cultura latinoamericana).

2. W. Week-end en Guatemala. La Habana: Ediciones La Tertulia, I960.

3. W. Week-end ent Guatemala. La Habana: Imprenta Nacional de Cuba, 1960. I94 p. (Ediciones especiales, 3).

4. Azuela, Mariano. Los de abajo. La Habana: Ediciones La Tertulia, 1960. (Biblioteca de cultura latinoamericana).

5. Balzac, Honorato de. La mujer de treinta años; novela. La Habana: Editorial Guerrero, I962.

6. - Papa Goriot. La Habana: Impr. Nacional de Cuba, I962. (Biblioteca del pueblo).

7. Baroja y Nessi, Pío. Aurora roja. Prólogo de Manuel Díaz Martínez. La Habana: Editorial Nacional de Cuba. Consejo Editora Nacional, I965. (Biblioteca del pueblo).

8. Beyle, Marie Henri. El rojo y el negro; crónica del siglo Xxx. La Habana: Editorial Nacional de Cuba, I962. 2 t. (Biblioteca del pueblo).

9. Blasco Ibáñez, Vicente. La barraca. La Habana: Editorial Nacional de Cuba, 196 .

Io. Bobadilla, Emilio. A fuego lento. Fray Candil en una novela, por Salvador Bueno. La Habana: Universidad de La Habana, r965. (Biblioteca de autores cubanos, no. 3r. Obras de Emilio Bobadilla Fray Candil, v. 3).

II. Brontë, Emily Jane. Cumbres borrascasas. La Habana: Impr. Mercaderes, 1962.

I2. Carpentier, Alejo. Los pasos perdidos. La Habana: Ediciones La Tertulia, 1960. (Biblioteca básica de cultura cubana, no. 29). 
13. Carrión, Miguel de. Las impuras. La Habana: Organización Continental de los festivales cel libro, r959. 2 t. (Biblioteca básica de cultura latinoamericana. Biblioteca básica de cultura cubana, ser. 2, I. 2-I3).

I4. Cervantes Saavedra, Miguel de. El ingenioso bidalgo Don Quijote de la Mancha. La Habana: Impr. Nacional, Gobierno revolucionario, r960. (Biblioteca del pueblo).

15. Daudet, Alfonso. Aventuras prodigtoscas de Tarlarin de Tarascón. La Habana: Impr. Nacional de Cuba, I962. (Biblioteca del pueblo).

r6. Delmar, Daniel. Coirón. La Habana: Editorial Nacional, I963.

17. Dickens, Charles John Huffman. Las memorias del Club Pickwick. La Habana: Impr. Nacional de Cuba, I962. (Biblioteca del pueblo).

I8. Dos Passos, John. Manbattan transfer. Prólogo de Lisandro Otero. La Habana: Editora del Consejo Nacional de Cultura, I965. (Colección biblioteca del pueblo).

19. Dumas, Alejandro. Los tres mosqueteros. La Habana: Editorial Nacional de Cuba, I965.

20. Fallas, Carlos Luis. Mamita Yunai. El infierno de las bananeras. La Habana: Organización Continental de los festivales del libro, r96r. (Biblioteca básica de cultura latinoamericana).

21. - Mamita Yunai; novela. La Habana: Impr. Nacional de Cuba, I962. (Ediciones especiales, no. 9).

22. Faulkner, William. Mientras agonizo. Prólogo de Luis Agüero. Traducción revisada de Ambrosio Fornet. La Habana: Editorial Nacional de Cuba. Editora del Consejo Nacional de Cultura, 1965. (Biblioteca del pueblo).

23. Fedin, Konstantin A. Primeras ale grias. La Habana: Impr. Nacional de Cuba, I962. (Biblioteca del pueblo).

24. Flaubert, Gustavo. Madame Bovary. La Habana: Editora del Consejo Nacional de Cultura, I965.

25. France, Anatole. El figón dle la reina Pantoja. La Habana: Editora del Consejo Nacional de Cultura, I963. (Biblioteca del pueblo).

26. Furmanov, Dmitir. Chapáev. La Habana: Impr. Nacional de Cuba, I96r. (Biblioteca del pueblo).

27. Gallegos, Rómulo. Doña Bárbara. La Habana: Organización Continenal de los Festivales de'l Libro, I959. (Biblioteca básica de cultura latinoamericana, 63).

28. Gogol, Nicolás V. Almas muertas. La Habana: Editora del Consejo Nacional de Cultura, I964. (Biblioteca' del pueblo). 
29. Gogol, Nicolás V. Taras Bulba. La Habana: Editora del Consejo Nacional de Cultura, I962.

30. Gómez de Avellaneda, Gertrudis. Sab; novela. La Habana: Editora del Consejo Nacional de Cultura, 1963.

3I. Goytisolo, Juan. La isla. La Habana: Ediciones R, I962.

32. Grossman, Vassili. El pueblo es inmortal. 2. ed. La Habana: Impr. Naçional de Cuba, I96r.

33. Güitaldes, Ricardo. Don Segundo Sombra. La Habana: Impr. Nacional de Cuba, I96r. (Serie Nuestra América).

34. Hemingway, Ernest. Adiós a las armas. Prólogo ce Armando Alvarez Bravo. La Habana: Editora del Consejo Nacional de Cultura, 1965. (Biblioteca del puebio).

35. - El viejo y el motr. Traducción autorizada por el autor, por Lino Novás Calvo. La Habana: Editora del Consejo Nacional de Cultura, I962.

36. Jesús, Carolina María. La favelc. La Habana: Casa de las Américas, 1965. (Colección literatura latinoamericana).

37. Joyce, James. Retuato del artista adolescente. Traducción revisada y prólogo de Edmundo Desnoes. La Habana: Editora Nacional de Cuba, I964. (Biblioteca del pueblo).

33. Kazakevich, E. El corazón de unt cmigo. La Habana: Editorial Nacional de Cuba, 1962. (Biblioteca del pueblo).

39. Koestler, Arthur. El cero y el infiniro. La Habana: Librerias Unidas, I96I.

4̧ㅇ. Lara, Jesús. Nwestra yawarnuncbi; novela quéchua. La Habana: Editora Nacional de Cuba, I962. (Biblioteca del pueblo).

4r. Lewis, Sinclair. Babbit. La Habana, I965. (Biblioteca del pueblo).

42. López Ubeda, Francisco. La piccrir Justina. La Habana: Consejo Nacional de Cultura, I963. (Biblioteca básica de cultura española).

43. London, Jack. El llamaldo de la seltus. La Habana: Impr. Nacional de Cuba, rg62. (Biblioteca del pueblo).

44. Loveira, Carlos. Generaltes y doctores. La Habana: Consejo Nacional de Cultura, Ministerio de Educación, I962.

45. - - Juan Crollo. La Habana: Ministerio de Educación, Consejo Nacional de Cultura, 1962. (Colección Novela). Incluye una entrevista con Loveira.

46. Malaparte, Curzio La piel. La Habana: Taller y oficina de Luz-Hilo, Impr. Económica Integral, Ig6r.

47. - Sangre. La Habana: Taller y Oficina de Luz-Hilo, Impr. Económica Integral, I96I, 
48. Malraux, André. La condición bumana. Prólogo ce Lisandro Otero. La Habana, I965.

49. Mann, Thomas. Mario y el mago. La Habana: Editora Nacional de Cuba, 1965 .

50. Maughan, William Somerset. El filo de la navaja; novela. La Habana: Editorial Guerrero, I962.

5r. Meza y Suárez Inclán, Ramón. El duelo de mi vecino; novela. La Habana: Comisión Nacional Cubana de la UNESCO, r96r.

52. Nguyen, Ngoc. Noup, héroe de las montañas. La Habana: Impr. Nacional de Cuba, 196r. (Biblioteca del pueblo).

53. Novás Calvo, Lino. El otro Cayo; novela, 2. ed. La Habana: Cruzada Latinoamericana de Difusión Cultural, r959. (Comprensión de Cuba, 2).

54. Orwell, George. 1894. [i. e. mil ochocientos noventa y cuatro]. La Habana: Editorial Librerías Unidas, I96r.

55. - La rebelión de la gramja. La Habana: Editorial Librerías Unidas, I96r.

56. Ostrovskii, Nikolai. Así se templó el acero; novela en dos partes. Traducción de J. Vento y A. Herraíz. La Habana: Impr. Nacional de Cuba, r96r. (Biblioteca del pueblo).

57. —. Nacidos de la tempestad. La Habana: Impr. Nacional de Cuba, rg6r. (Biblioteca del pueblo).

58. Pérez Galdós, Benito. Doña Perfecta. Prólogo de Herminio Almendros. La Habana: Editora del Consejo Nacional de Cultura, I965. (Biblioteca del pueblo).

59. - - El 2 [ 2 . e dos] de mayo. La Habana: Empresa Consolidada ce Artes Gráficas, Unidad 1205, 1962. (Biblioteca del pueblo).

6o. Planas y Saínz, Juan Manuel. El sargaza de oro. (El vellocino verde); novela. 2. ef. La Habana: Cía. Editora de libros y folletos, I959.

6r. Prieto, Jenaro. El socio. La Habana: Casa de las Américas, I964. (Colección literaria latinoamericana).

62. Proust, Marcel. Un amor de Swamn. Prólogo de Virgilio Piñera. Traducción revisada por Virgilio Piñera. La Habana: Editora del Consejo Nacional de Cultura, I964. (Biblioteca del pueblo).

63. Ramos, Graciliano. Vidas secas. La Habana: Eçitora Nacional de Cuba. Casa de las Américas, I964. (Colección literatura latinoamericana).

64. Ramos, José Antonio. Caniquí. La Habana: Organización Continental 
de los Festivales del Libro, I959. (Biblioteca básica de cultura latinoamericana, I05. Biblioteca básica de cultura cubana, ser. 2., I5).

65. Ramos, José Antonio. Caniqui; novela. 2. ed. La Habana: Editora del Consejo Nacional de Cultura, 1964. (Biblioteca de autores cubanos).

66. Rivera, José Eustacio. La voráginze. La Habana: Organización Continental de los Festivales del Libro, I959. (Biblioteca básica de cultura latinoamericana).

67. - - La vorágine. La Habana: Editorial Prensa Libre, I960.

68. —. La vorágine. La Habana: Editorial Lex, I960.

69. Rodríguez, Luis Felipe. La conjura de la ciénaga. La Habana: Organización Continental de los Festivales del Libro, I959. (Biblioteca básica de cultura latinoamericana, II6. Biblioteca básica de cultura cubana. ser. 2., I7).

70. Rolland, Romain. Iuan Cristóbal. La Habana: Editorial Nacional de Cuba, 1963. (Biblioteca del pueblo).

71. Romero, Rubén. La vida inuitil de Pito Pérez. La Habana: Editora Nacional de Cuba, I964. (Colección literatura latinoamericana II).

72. Sholojov, Mijail. El Don apaciblle. La Habana: Empresa Consolidada de Artes Gráficas, I962. (Biblioteca del pueblo).

73. - - El Don arrasado. La Habana: Editora Nacional de Cuba, I963. (Biblioteca del pueblo).

74. - Sangre els el Don. La Habana: Editora del Consejo Nacional de Cultura, I962. (Biblioteca del pueblo).

75. - - Sie desborda el Don. La Habana: Editora del Consejo Nacional de Cultura, I962. (Biblioteca del pueblo).

76. Solzhentsin, Alexande. Un dia de Ivär Dentsavich. La Habana: Editorial Nacional de Cuba. Editora del Consejo Nacional, r965. (Colección Cocuyo. Literatura Universal).

77. Stowe, Harriet Beecher. La cabañ del Tía Tom. La Habana: Editora Nacional de Cuba, Ig64. (Editora Juvenil).

78. Tendriakov, Vladimir Fedorovich. En casa djena. La Habana: Tierra nueva, I96r. (Festival de literatura soviética).

79. Tolstoi, Lcón Nicolás. Ana Karenina. La Habana: Impr. Nacional de Cuba, I962. (Obras maestras, I).

8o. - - Resumección; novela. La Habana: Editorial Guerrero, I962.

8I. Turgenev, Iván. Nido de bidalgos. La Habana: Impr. Nacional de Cuba, I962. (Biblioteca del pueblo).

82. Steinbeck, John. Las viñas de la ira. La Habana: Editora del Consejo Nacional de Cultura, r963. (Biblioteca del pueblo). 
83. Valle Inclán, Ramón. Tirano Banderas; novela de tierra caliente. La Habana: Editorial Nacional de Cuba, Editora del Consejo Nacional de Cultura, r964. (Biblioteca básica de literatura española).

84. Vasov, Iván. Bajo el yugo; novela. 1876. La Habana: Editora del Consejo Nacional de Cultura, I963. (Traducción del búlgaro por T. Neicov).

85. Vida del Lazwillo de Tormes y de sus fortunas y adversidades. La Habana: Impr. Nacional de Cuba, I962. (Biblioteca del pueblo).

86. Villaverde, Cirilo. Cecilia Valdés; novela de costumbres cubanas. La Habana: Organización Continental de Festivales del Libro, I959. (Biblioteca básica de cultura latinoamericana, IOI).

87. —. Cécilia Valdés. La Habana: Editorial Nacional de Cuba, Editora del Consejo Nacional de Cultura, I964. (Biblioteca básica de autores cubanos).

88. - La joven de la flecha de oro. Prólogo de Mario Parajón. La Habana: Comisión Nacional Cubana de la UNESCO, r962.

89. —- La peinleta calada. La Habana: Comisión Nacional Cubana de la UNESCO, I962.

90. - La tiejedora de sombreros de yarey. La Habana: Comisión Nacional Cubana de la UNESCO, I962.

9r. Voltaire, Francois Marie Arnault de. Cándido o El optimista. La Habana: Impr. Nacional de Cuba, I96I.

92. West, Nathanael. Srta. Corazones Solitarios. La Habana: Ediciones $\mathrm{R}, \mathrm{rg} 6_{3}$.

Rosa Abella

University of Miami Library 